\title{
Glutathione Biosynthesis via Activation of the Nuclear Factor E2- Related Factor 2 (Nrf2) - Antioxidant-Response Element (ARE) Pathway Is Essential for Neuroprotective Effects of Sulforaphane and 6-(Methylsulfinyl) Hexyl Isothiocyanate
}

\author{
Keita Mizuno ${ }^{1}$, Toshiaki Kume ${ }^{1}$, Chie Muto ${ }^{1}$, Yuki Takada-Takatori ${ }^{2}$, Yasuhiko Izumi ${ }^{1}$, Hachiro Sugimoto ${ }^{3}$, \\ and Akinori Akaike ${ }^{1, *}$ \\ ${ }^{1}$ Department of Pharmacology, ${ }^{3}$ World-Leading Drug Discovery Research Center, \\ Graduate School of Pharmaceutical Sciences, Kyoto University, 46-29 Shimoadachi-cho, Sakyo-ku, Kyoto 606-8501, Japan \\ ${ }^{2}$ Department of Pharmacology, Faculty of Pharmaceutical Sciences, Doshisha Women's College, Kyoto 610-0395, Japan
}

Received October 8, 2010; Accepted January 4, 2011

\begin{abstract}
Oxidative stress plays pivotal roles in aging, neurodegenerative disease, and pathological conditions such as ischemia. We investigated the effect of sulforaphane and 6-(methysulfinyl) hexyl isothiocyanate (6-HITC), a naturally occurring isothiocyanate, on oxidative stressinduced cytotoxicity using primary neuronal cultures of rat striatum. Pretreatment with sulforaphane and 6-HITC significantly protected against $\mathrm{H}_{2} \mathrm{O}_{2}$ - and paraquat-induced cytotoxicity in a concentration-dependent manner. Sulforaphane and 6-HITC induced the translocation of nuclear factor E2-related factor 2 (Nrf2) into the nucleus and increased the expression of $\gamma$-glutamylcysteine synthetase ( $\gamma$-GCS), a rate-limiting enzyme in glutathione synthesis, and the intracellular glutathione content. Treatment with reduced glutathione (GSH) and $N$-acetyl-L-cysteine, a substance for glutathione synthesis, significantly prevented the cytotoxicity induced by $\mathrm{H}_{2} \mathrm{O}_{2}$ and paraquat. Moreover, exposure to L-buthionine-sulfoximine, an irreversible inhibitor of $\gamma$-GCS, suppressed the protective effects of sulforaphane and 6-HITC. In contrast, sulforaphane and 6-HITC increased heme oxygenase-1 (HO-1) expression in neurons. However, zinc-protophorphyrin IX, a competitive inhibitor of HO-1, did not influence the protective effects of sulforaphane and 6-HITC. These results suggest that sulforaphane and 6-HITC prevent oxidative stress-induced cytotoxicity in rat striatal cultures by raising the intracellular glutathione content via an increase in $\gamma$-GCS expression induced by the activation of the Nrf2-antioxidant response element pathway.
\end{abstract}

Keywords: isothiocyanate, neuroprotection, oxidative stress, nuclear factor E2-related factor 2 (Nrf2)

\section{Introduction}

Oxidative stress has been implicated in aging and the pathogenesis of various neurodegenerative disorders such as Alzheimer's disease, Parkinson's disease, and Huntington's disease. Oxidative stress results in an imbalance of pro-oxidant/antioxidant homeostasis that leads to the generation of reactive oxygen species (ROS), which are highly toxic to cells. The brain is particularly

*Corresponding author. aakaike@pharm.kyoto-u.ac.jp Published online in J-STAGE on February 24, 2011 (in advance) doi: 10.1254/jphs. 10257FP susceptible to the damage by ROS because of its high metabolic rate and relatively reduced capacity for restoration compared with other organs $(1-4)$. The antioxidant-response element (ARE) is a cis-acting DNA element essential for transcriptional activation of phase-II genes such as those encoding UDP-glucuronosyl transferase and glutathione-S-transferase and the genes for antioxidants such as NADPH: quinone reductase and heme oxygenase-1 (HO-1). The activation of ARE is dependent on the translocation of nuclear factor E2related factor 2 (Nrf2) into the nucleus through its release from kelch-like ECH-associated protein 1 (Keap1), the cytoplasmic repressor of Nrf2. Nrf2 binds to ARE in the 
nucleus and promotes the transactivation of the AREcoded genes $(5,6)$.

Isothiocyanates are contained in cruciferous vegetables such as broccoli, Brussel sprouts, cauliflower, and cabbage. Sulforaphane, which is found at high levels in broccoli sprouts and broccoli (7), and 6-(methylsulfinyl) hexyl isothiocyanate (6-HITC), which is contained in wasabi (8), are naturally occurring isothiocyanates. All these compounds have an isothiocyanate group (NCS) (Fig. 1: A, B). Sulforaphane and 6-HITC are known to be potent Nrf2 activators and exhibit anti-oxidative and anti-carcinogenic effects via the up-regulation of AREdriven genes $(9-12)$.

Isothiocyanates have beneficial effects in various types of cells derived from peripheral tissues. Moreover, several studies have reported that sulforaphane has protective effects on neurons of the central nervous system $(13-15)$. However, there are few studies on the detailed mechanism of neuroprotective effects of isothiocyanates in the central nervous system. In the present study, we examined the effects of sulforaphane and 6-HITC on oxidative stress-induced cytotoxicity using primary neuronal cultures of rat striatum.

\section{Materials and Methods}

\section{Drugs and chemicals}

Eagle's minimum essential medium (Eagle's MEM) was purchased from Nissui Pharmaceutical (Tokyo). Fetal bovine serum was purchased from JRH Bioscience (Lenexa, KS, USA). Sulforaphane and 6-HITC were obtained from LKT Laboratory (St. Paul, MN, USA).
A

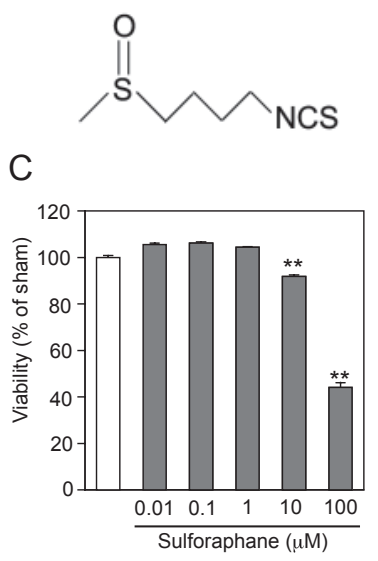

B

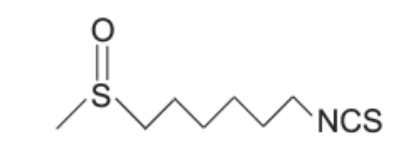

D

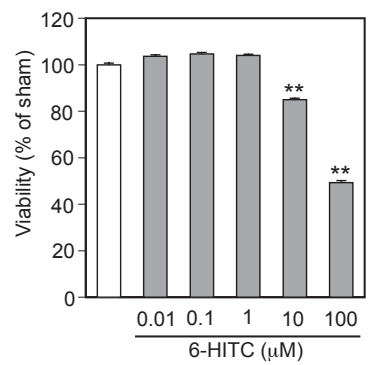

Fig. 1. Effects of sulforaphane and 6-HITC on viability of rat striatal cultures. Chemical structure of sulforaphane (A) and 6-HITC (B). Cultures were treated with $0.01-100 \mu \mathrm{M}$ sulforaphane (C) or $0.01-100 \mu \mathrm{M} 6$-HITC (D) for $48 \mathrm{~h}$. ${ }^{* *} P<0.01$, compared with sham-treatment.
5,5'-Dithiobis-(2-nitrobenzonic acid) (DTNB), NADPH, dimethylsufoxide, glucose, glutamine, HEPES, and $\mathrm{NaHCO}_{3}$ were from Nacalai Tesque (Kyoto). L-Buthionine- $[S, R]$-sulfoximine (BSO), $N$-acethyl-L-cysteine (NAC), and 1.1'-dimethyl-4,4'-bipyridinium dichloride (paraquat) were obtained from Sigma (St. Louis, MO, USA). Reduced glutathione (GSH) and hydrogen peroxide $\left(\mathrm{H}_{2} \mathrm{O}_{2}\right)$ were from Wako Pure Chemicals (Osaka). Zinc-protoporphyrin IX (Zn-PP IX) was obtained from Calbiochem (San Diego, CA, USA). Glutathione reductase was from Roche Diagnostics (Basel, Switzerland). The cytotoxicity detection lactate dehydrogenase (LDH) kit was purchased from Kyokuto Pharmaceutical Industrial Corp. (Tokyo).

\section{Cell culture}

The animals were treated in accordance with the guidelines of the Kyoto University animal experimentation committee and the guidelines of The Japanese Pharmacological Society. Primary cultures were prepared from the anterior striatum of fetal Wistar rats (Nihon SLC, Shizuoka) at the 17 th -19 th days of gestation, according to previously described procedures (16). Briefly, the anterior striatum of fetal rats was removed bilaterally, mechanically dissociated using scalpel blades, and then filtered through a stainless steel mesh. Single-cell suspensions were placed on $0.1 \%$ polyethylenimine-coated 24-well plates (Falcon, Franklin Lakes, NJ, USA) or $35-\mathrm{mm}$ dishes at a density of $4.0-4.1 \times 10^{5}$ cells $/ \mathrm{cm}^{2}$. Cultures were maintained in Eagle's MEM supplemented with $10 \%$ heat-inactivated fetal bovine serum, glutamine $(2 \mathrm{mM})$, glucose (total $11 \mathrm{mM}), \mathrm{NaHCO}_{3}(24 \mathrm{mM})$, and HEPES $(10 \mathrm{mM})$ at $37^{\circ} \mathrm{C}$ in a humidified atmosphere of $5 \% \mathrm{CO}_{2}$ and $95 \%$ air.

\section{Drug treatment}

Cultures were used at $3-5$ DIV for this study. At 3 days after plating, cultures were incubated in the presence or absence of sulforaphane, 6-HITC, GSH, NAC, or $\mathrm{BSO}$ for $24 \mathrm{~h}$. Cultures were exposed to paraquat or $\mathrm{H}_{2} \mathrm{O}_{2}$ for $24 \mathrm{~h}$ at $4 \mathrm{DIV}$. After drug treatment, cell viability was determined with a LDH release assay. In each experiment, cells in four wells received drug treatment to obtain the mean \pm S.E.M. of cell viability. The reproducibility of the results was confirmed with at least three sets of experiments.

\section{$L D H$ release assay}

Cell viability was assessed by measuring the amount of LDH released into the culture medium using a Cytotoxicity Detection LDH kit as described previously (16). Briefly, the culture supernatant was added to the LDH substrate mixture. After incubation at $37^{\circ} \mathrm{C}$, the reaction 
was stopped by adding $1 \mathrm{~N} \mathrm{HCl}$ and the absorbance was measured at $570 \mathrm{~nm}$ with a microplate reader (Bio-Rad, Hercules, CA, USA). The reading of background absorbance, consisting of culture medium and each drug in the absence of cells, was subtracted from each value. Cell viability was evaluated relative to the total amount of $\mathrm{LDH}$ released by exposure to $1 \mathrm{mM} \mathrm{H}_{2} \mathrm{O}_{2}$ for $24 \mathrm{~h}$.

\section{Measurement of glutathione content}

Total glutathione (reduced and oxidized glutathione) content was measured according to the methods described previously (17). Briefly, cultures were washed with Trisbuffered saline on ice and then deproteinated with $10 \%$ trichloroacetic acid. Samples were centrifuged, and the supernatant was mixed with $200 \mathrm{mM}$ phosphate-buffered saline, $0.5 \mathrm{mM}$ EDTA, and $0.4 \mathrm{mM}$ NADPH. Glutathione reductase was added and incubated for $10 \mathrm{~min}$. Subsequently, the reaction was initiated by adding $10 \mathrm{mM}$ DTNB and the mixures were incubated for $10 \mathrm{~min}$. The absorbance was measured at $450 \mathrm{~nm}$ with a microplate reader. Total glutathione content was determined with a standard curve obtained from defined concentrations of reduced glutathione.

\section{Preparation of the nuclear extract}

To obtain nuclear proteins, striatal cells were washed with cold Tris-buffered saline on ice, resuspended in a 10 mM HEPES ( $\mathrm{pH}$ 7.9) buffer containing $10 \mathrm{mM} \mathrm{KCl}, 0.1$ mM EGTA, 0.5\% Nonidet P-40, $1 \mathrm{mM}$ vanadate, $1 \%$ aprotinin, $1 \mathrm{mM}$ dithiothreitol, and $1 \mathrm{mM}$ phenylmethylsulfonyl fluoride and incubated on ice for $15 \mathrm{~min}$. After centrifugation at 3,000 rpm for $5 \mathrm{~min}$, the resulting nuclear pellet was resuspended in $20 \mathrm{mM}$ HEPES ( $\mathrm{pH}$ 7.9) containing $420 \mathrm{mM} \mathrm{NaCl}, 1 \mathrm{mM}$ EGTA, $1 \mathrm{mM}$ EDTA, $1 \mathrm{mM}$ dithiothreitol, and $1 \mathrm{mM}$ phenylmethylsulfonyl fluoride; extracted on ice for $30 \mathrm{~min}$; and centrifuged at $14,000 \mathrm{rpm}$ for $10 \mathrm{~min}$. The supernatant was collected as the nuclear extract.

\section{Western blot analysis}

After treatment with drugs for the indicated periods, cells were washed twice with cold Tris-buffered saline on ice and harvested using a cell scraper. Cells were lysed in a $20 \mathrm{mM}$ Tris buffer ( $\mathrm{pH} \mathrm{7)}$ containing $25 \mathrm{mM}$ $\beta$-glycerophosphate, 2 mM EGTA, $1 \%$ Triton X-100, 1 $\mathrm{mM}$ vanadate, $1 \%$ aprotinin, $1 \mathrm{mM}$ phenylmethylsulfonyl fluoride, and $2 \mathrm{mM}$ dithiothreitol. The lysate was centrifuged at 15,000 rpm for $30 \mathrm{~min}$, and the supernatant was denatured by boiling for $5 \mathrm{~min}$. Aliquots of protein were loaded on a sodium dodecyl sulfate-polyacrylamide gel, separated electrophoretically, and transferred to a polyvinylidene fluoride microporous membrane (Millipore, Corp., Bedford, MA, USA). After blocking with non-fat milk, the membrane was incubated with primary antibodies [anti- $\gamma$-gultamylcysteine synthetase $(\gamma$-GCS), antiheme oxygenase-1 (HO-1), anti-lamin B (Santa Cruz Biotechnology, Inc., Santa Cruz, CA, USA), anti-Nrf2 (Cell Signaling Technology, Inc., Danvers, MA, USA), and anti- $\beta$-actin (Sigma)] and with a secondary antibody. Immunodetection was performed using the ECL Western blotting detecting system (Amersham Biosciences UK Ltd., Buckinghamshire, UK).

\section{Immunocytochemistry}

After drug treatment, cells were fixed with phosphatebuffered saline (PBS) containing 4\% paraformaldehyde for $15 \mathrm{~min}$ at room temperature, washed three times with PBS, and blocked with $0.2 \%$ Triton X-100 in PBS for 30 min. Cultures were incubated at $4^{\circ} \mathrm{C}$ overnight with the primary antibodies [anti-microtubule-associated protein $(2 \mathrm{a}+2 \mathrm{~b})(\mathrm{MAP} 2 \mathrm{ab})($ Sigma), and anti-HO-1 antibody]. After three rinses with PBS, the cultures were incubated for $90 \mathrm{~min}$ at room temperature with secondary antibodies diluted in $0.2 \%$ Triton X-100 in PBS: Alexa Flour 488-labeled goat anti-mouse IgG $(\mathrm{H}+\mathrm{L})$ (Molecular Probes, Eugene, OR, USA) and Alexa Flour 594-labeled goat anti-rabbit IgG $(\mathrm{H}+\mathrm{L})$ (Molecular Probes). Immnofluorescence was visualized with a fluorescence microscope (Leica, Wetzlar, Germany).

\section{Statistical analyses}

Data were expressed as the mean \pm S.E.M. The reproducibility of the results was confirmed in at least three different sets of experiments. All statistical analyses were performed with GraphPad Instat version 3.0 (GraphPad Software, Inc., San Diego, CA, USA). The statistical significance of the difference between groups was calculated by a one-way analysis of variance and post hoc multiple comparison using Dunnett's test. A difference was considered significant at $P<0.05$.

\section{Results}

Effects of sulforaphane and 6-HITC on viability of rat striatal cultures

We examined whether sulforaphane and 6-HITC affect the survival of rat striatal cultures. The viability of striatal cultures was not affected by treatment with sulforaphane at $0.01-1 \mu \mathrm{M}$ or 6 -HITC at $0.01-1 \mu \mathrm{M}$ for $48 \mathrm{~h}$. However, sulforaphane and 6-HITC at concentrations of more than $10 \mu \mathrm{M}$ caused cell death (Fig. 1: C, D).

Effects of sulforaphane and 6-HITC on oxidative stressinduced cytotoxicity in rat striatal cultures

We examined the effect of sulforaphane and 6-HITC on oxidative stress-induced cytotoxicity. In this experi- 
ment, we utilized paraquat and $\mathrm{H}_{2} \mathrm{O}_{2}$ to generate various types of oxidative stress. Paraquat reacts with NADPH to produce the paraquat radical and also reacts with $\mathrm{O}_{2}$ to generate $\mathrm{O}_{2}^{-\cdot}(18,19) . \mathrm{O}_{2}^{--}$is immediately dismutated into $\mathrm{H}_{2} \mathrm{O}_{2}$. In turn, $\mathrm{H}_{2} \mathrm{O}_{2}$ exerts its toxic effects mainly through the ferrous iron-dependent formation of highly reactive $\mathrm{OH}$. Exposure to paraquat and $\mathrm{H}_{2} \mathrm{O}_{2}$ for $24 \mathrm{~h}$ reduced the cell viability of striatal cultures (Fig. 2). Sulforaphane $(0.01-1 \mu \mathrm{M})$ and 6-HITC $(0.01-1 \mu \mathrm{M})$ administered for $24 \mathrm{~h}$ before exposure to the toxic agents, significantly attenuated the cytotoxicity induced by paraquat $(200 \mu \mathrm{M})$ and $\mathrm{H}_{2} \mathrm{O}_{2}(30 \mu \mathrm{M})$ in a concentrationdependent manner (Fig. 2: A - D). However, co-application of sulforaphane and 6-HITC with paraquat and $\mathrm{H}_{2} \mathrm{O}_{2}$ for $24 \mathrm{~h}$ did not affect the cytotoxicity (data not shown).

\section{Sulforaphane and 6-HITC induce the translocation of} Nrf2 into the nucleus

Several lines of evidence indicate that isothiocyanates stimulate the translocation of Nrf2, a member of the basic leusine zipper transcription factor family, into the nucleus, and then enhances the transactivation of ARE-encoded genes $(8,20,21)$. To investigate the neuroprotective mechanisms of sulforaphane and 6-HITC, we examined

A
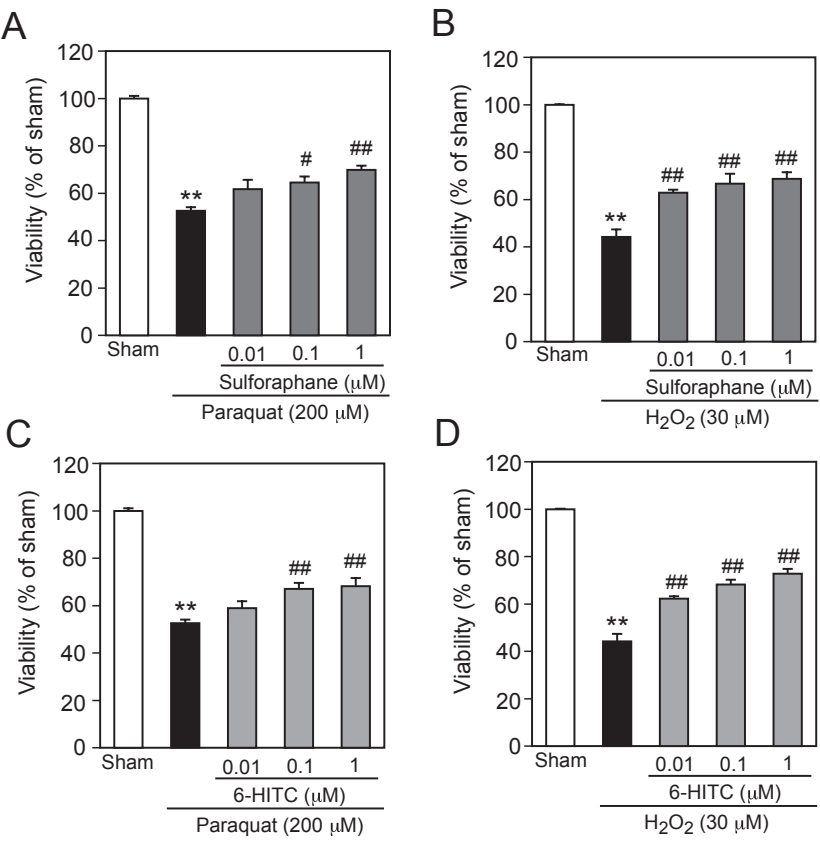

Fig. 2. Protective effects of isothiocyanates on oxidative stress-induced cytotoxicity in rat striatal cultures. Cultures were pre-treated with $0.01-1 \mu \mathrm{M}$ sulforaphane for $24 \mathrm{~h}$ and simultaneously exposed to $200 \mu \mathrm{M}$ paraquat (A) and $30 \mu \mathrm{M} \mathrm{H}_{2} \mathrm{O}_{2}$ (B) for $24 \mathrm{~h}$. Cultures were pre-treated with $0.01-1 \mu \mathrm{M} 6$-HITC for $24 \mathrm{~h}$ and concurrently treated with $200 \mu \mathrm{M}$ paraquat (C) and $30 \mu \mathrm{M} \mathrm{H}_{2} \mathrm{O}_{2}$ (D) for $24 \mathrm{~h}$. ${ }^{* *} P<0.01$, compared with sham-treatment; ${ }^{\#} P<0.05,{ }^{\#} P<0.01$, compared with toxin alone. the effect of sulforaphane and 6-HITC on the translocation of Nrf2 into the nucleus. As shown in Fig. 3A, sulforaphane increased the amount of $\mathrm{Nrf} 2$ after $0.5 \mathrm{~h}$ in the nuclear fraction. Exposure to 6-HITC also stimulated a translocation of Nrf2 into the nucleus after $1 \mathrm{~h}$ (Fig. 3B).

Sulforaphane and 6-HITC stimulated the expression of $\gamma-G C S$ and increased glutathione contents in striatal cultures

The tripeptide glutathione is the most abundant thiol present in mammalian cells and is important for cellular defense against ROS as an antioxidant $(22,23)$. It has been reported that $\gamma$-GCS is activated by the binding of Nrf2 to the ARE $(5,20)$. To determine the neuroprotective mechanism of sulforaphane and 6-HITC against oxidative stress, we examined the effect on the expression of $\gamma$-GCS by western blotting. After treatment with sulforaphane $(0.01-10 \mu \mathrm{M})$ and 6-HITC $(0.01-10 \mu \mathrm{M})$, the expression level of $\gamma$-GCS was increased in a concentration-dependent manner (Fig. 4A). We next examined whether sulforaphane and 6-HITC increase the amount of intracellular glutathione. Exposure to $1 \mu \mathrm{M}$ sulforaphane and $1 \mu \mathrm{M}$ 6-HITC significantly increased the total glutathione (reduced and oxidized glutathione) content (Fig. 4: B, C).

Involvement of glutathione in cytoprotection by sulforaphane and 6-HITC against oxidative stress

As sulforaphane and 6-HITC increased the amount of glutathione in a time-dependent manner, we investigated the involvement of glutathione in the cytoprotection by sulforaphane and 6-HITC. The administration of GSH at $30-300 \mu \mathrm{M}$ for $24 \mathrm{~h}$ before exposure to $\mathrm{H}_{2} \mathrm{O}_{2}$ or paraquat significantly attenuated cytotoxicity in a concentrationdependent manner (Fig. 5: A, C). Furthermore, pretreat-

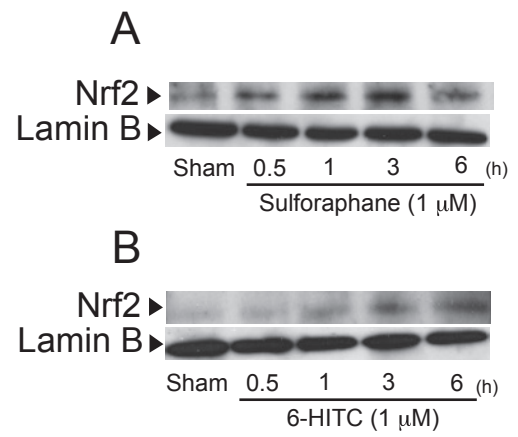

Fig. 3. Effect of isothiocyanates on nuclear translocation of Nrf2 in rat striatal cultures. The nuclear fraction separated from isothiocyanate-treated cells was subjected to immunoblotting for the detection of Nrf2 and lamin B. Cultures were treated with $1 \mu \mathrm{M}$ sulforaphane (A) and $1 \mu \mathrm{M}$ 6-HITC (B) for the indicated periods. Lamin B is a loading control. 
A
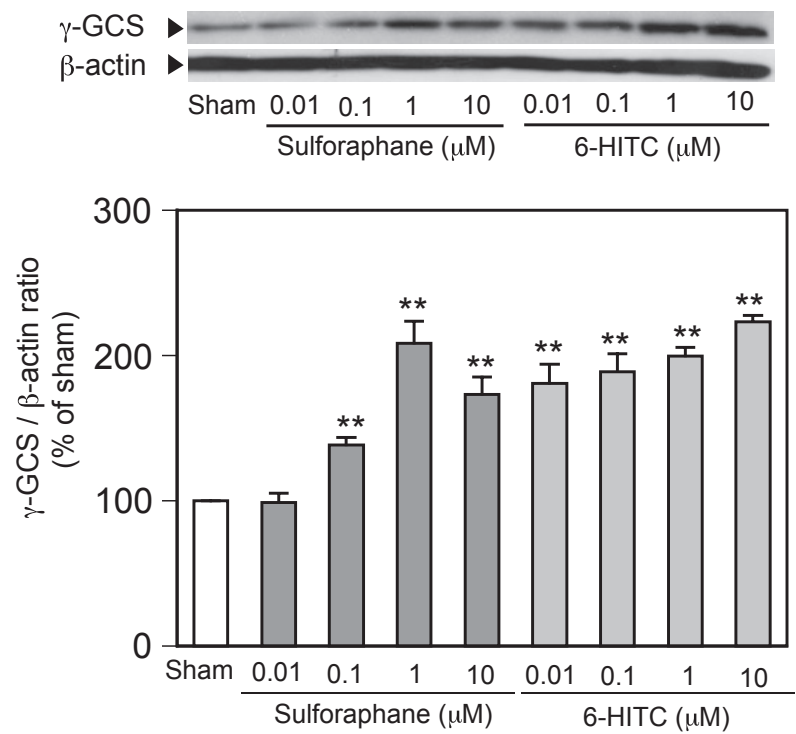

B

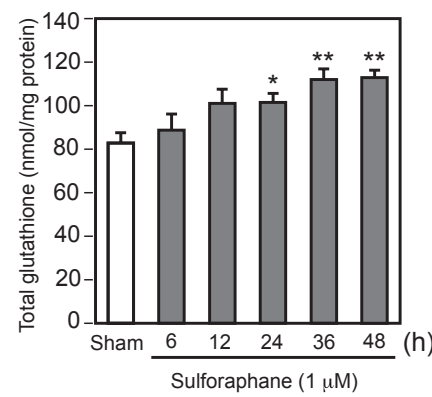

C

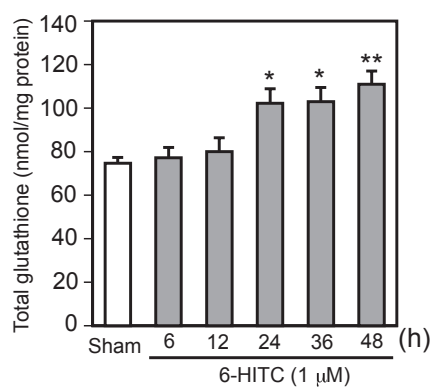

Fig. 4. Effects of isothiocyanates on expression of $\gamma$-GCS and the total amount of glutathione (GSH and GSSG) in rat striatal cultures. A: The protein levels of $\gamma$-GCS and $\beta$-actin in cell lysate were determined by immunoblotting. Cultures were treated with $0.01-10 \mu \mathrm{M}$ sulforaphane and $0.01-10 \mu \mathrm{M}$ 6-HITC for $12 \mathrm{~h}$. B, $\mathrm{C}$ : Total amount of glutathione following treatment with $1 \mu \mathrm{M}$ sulforaphane and $1 \mu \mathrm{M}$ 6-HITC for the indicated periods. $* P<$ $0.05, * * P<0.01$, compared with sham-treatment.
A

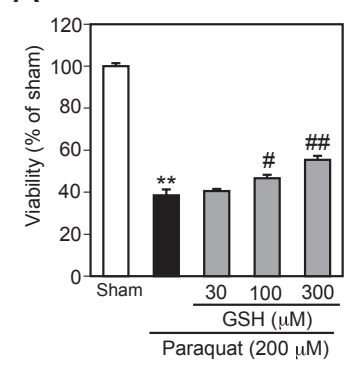

D

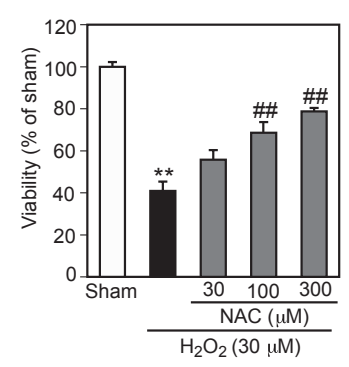

G

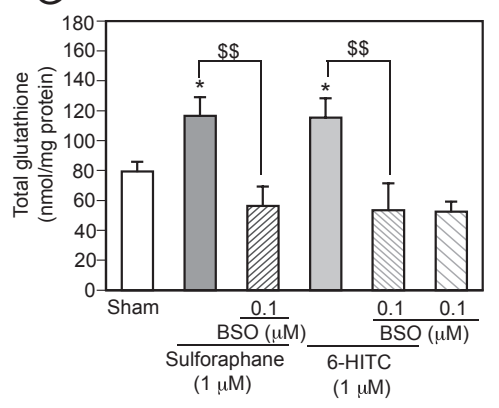

B

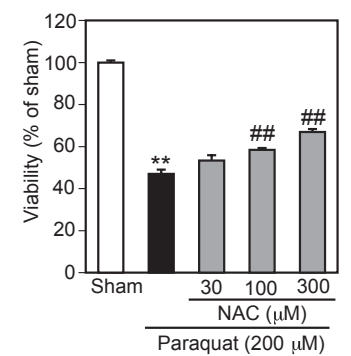

$\mathrm{E}$

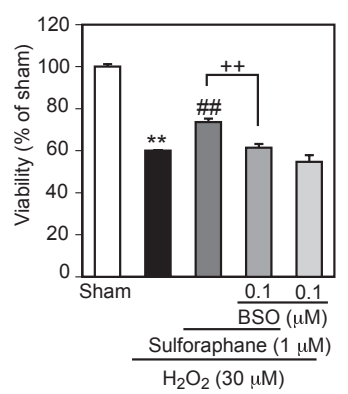

C

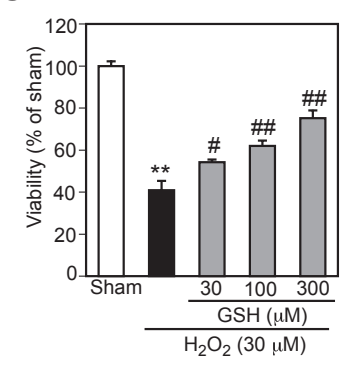

$\mathrm{F}$

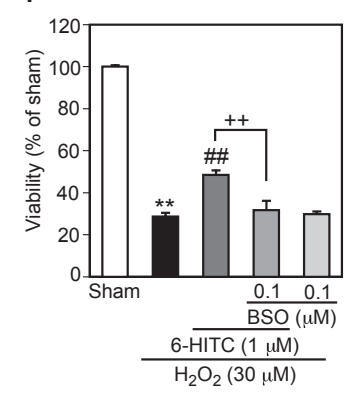

Fig. 5. Involvement of glutathione in the protective effect of isothiocyanates on oxidative stressinduced cytotoxicity. A, B: Cultures were pre-treated with $30-300 \mu \mathrm{M}$ GSH (A) and $30-300 \mu \mathrm{M}$ NAC (B) for $24 \mathrm{~h}$ and then exposed to $200 \mu \mathrm{M}$ paraquat for $24 \mathrm{~h}$. C, D: Cultures were pre-treated with $30-300 \mu \mathrm{M}$ GSH (C) and $30-300 \mu \mathrm{M}$ NAC (D) for $24 \mathrm{~h}$ and then treated with $30 \mu \mathrm{M} \mathrm{H}_{2} \mathrm{O}_{2}$ for $24 \mathrm{~h}$. E, F: BSO was pre-treated with sulforaphane (E) and 6-HITC (F) for $24 \mathrm{~h}$, and then exposed to $30 \mu \mathrm{M}$ $\mathrm{H}_{2} \mathrm{O}_{2}$ for $24 \mathrm{~h}$. G: Total amount of glutathione following treatment with $0.1 \mu \mathrm{M}$ BSO and $1 \mu \mathrm{M}$ sulforaphane or $1 \mu \mathrm{M}$ 6-HITC for $24 \mathrm{~h} .{ }^{*} P<0.05,{ }^{*} P P<0.01$, compared with sham-treatment; ${ }^{*} P<0.05$, ${ }^{\#} P<0.01$, compared with toxin alone; ${ }^{+} P<0.01$, compared with isothiocyanates and toxin; ${ }^{s s} P<0.01$. 
ment with NAC $(30-300 \mu \mathrm{M})$, a precursor for glutathione synthesis, for $24 \mathrm{~h}$ significantly blocked the cytotoxicity induced by $\mathrm{H}_{2} \mathrm{O}_{2}$ and paraquat (Fig. 5: B, D). We next examined the effect of BSO, an irreversible inhibitor of $\gamma$-GCS, on cytoprotection by sulforaphane and 6-HITC. The co-administration of $0.1 \mu \mathrm{M}$ BSO significantly suppressed the protective effects of sulforaphane and 6-HITC (Fig. 5: E, F). In addition, $0.1 \mu \mathrm{M}$ BSO suppressed the intracellular glutathione content increased by sulforaphane and 6-HITC (Fig. 5G).
Sulforaphane and 6-HITC increase the expression of HO-1 in neurons

Previous studies have demonstrated that HO-1 expression is up-regulated via the activation of the Nrf2-ARE pathway $(5,24,25)$. We next examined whether sulforaphane and 6-HITC increase the expression of HO-1 in primary striatal cultures. As shown in Fig. 6: A, B, treatment with $1 \mu \mathrm{M}$ sulforaphane or $1 \mu \mathrm{M} 6$-HITC increased the expression of HO-1, which peaked at $12 \mathrm{~h}$ after treatment. To determine whether de novo protein synthesis is required for the increased expression of
A
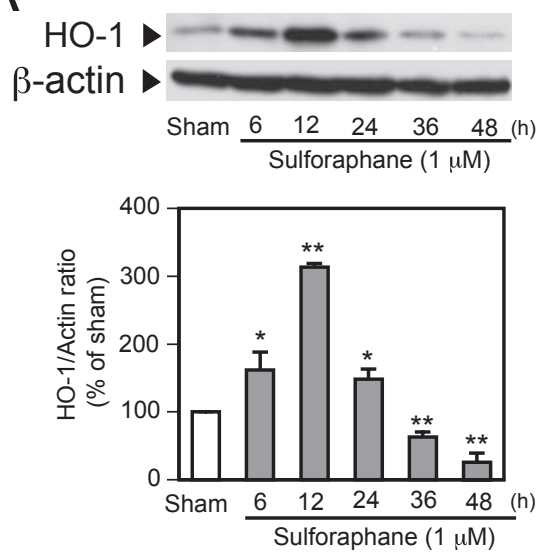

C

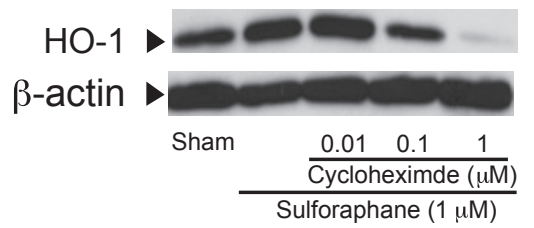

E
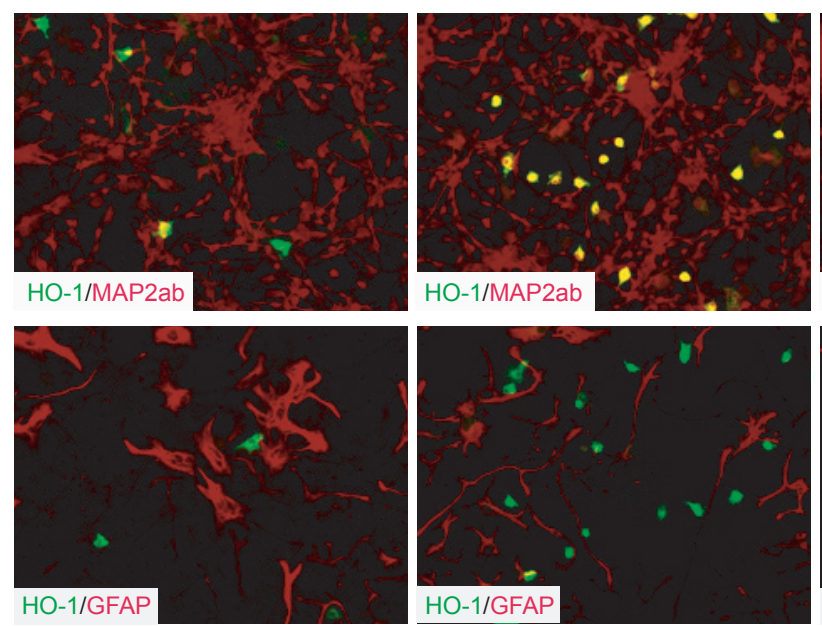

Sham

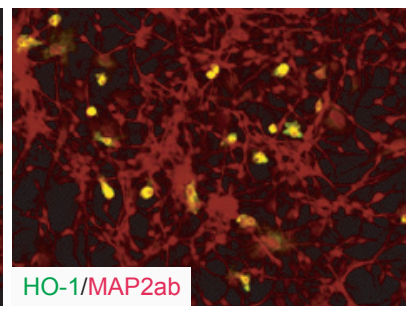

B
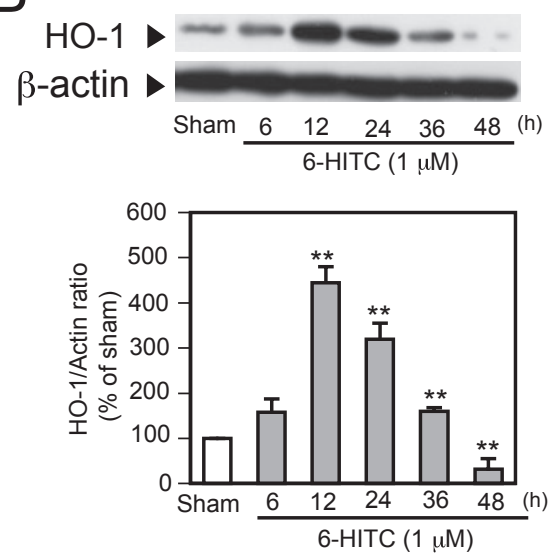

D
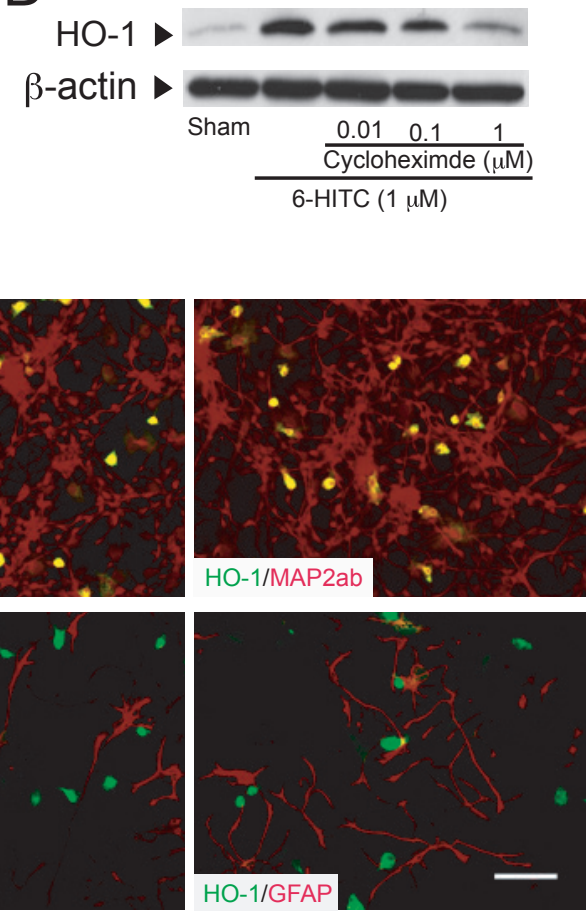

6-HITC

$(1 \mu \mathrm{M})$
Fig. 6. Effect of isothiocyanates on expression of HO-1 in rat stiratal cultures. The protein levels of HO- 1 and $\beta$-actin in cell lysates were determined by immunoblotting. A, B: Cultures were treated with $1 \mu \mathrm{M}$ sulforaphane (A) and 1 $\mu \mathrm{M} 6$-HITC (B) for the indicated periods. $* P<0.05, \quad * * P<0.01$, compared with sham-treatment. C, D: Cycloheximide at $0.01-1 \mu \mathrm{M}$ was simultaneously applied with 1 $\mu \mathrm{M}$ sulforaphane (C) and $1 \mu \mathrm{M}$ 6-HITC (D) for 12 h. E: Representative microphotographs of HO-1 expression. Cultures were treated with $1 \mu \mathrm{M}$ sulforaphane and $1 \mu \mathrm{M}$ 6-HITC for $12 \mathrm{~h}$. Cells were fixed and immunostained with antiHO-1, anti-MAP2ab, and antiGFAP antibodies. Neurons and astrocytes were identified by the marker proteins MAP2ab and GFAP. Scale bar: $50 \mu \mathrm{m}$. 
HO-1, cycloheximide, an inhibitor of cytoplasmic protein synthesis, was simultaneously applied with sulforaphane and 6-HITC. Cycloheximide $(0.01-1 \mu \mathrm{M})$ reduced HO-1 expression induced by sulforaphane and 6-HITC in a concentration-dependent manner (Fig. 6: C, D). Furthermore, to identify the type of cells that increased the expression of HO-1 by sulforaphane and 6-HITC, we employed immunofluorescence staining with antiMAP2ab, a neuronal marker protein, and anti-GFAP, a marker of astrocytes. Exposure to sulforaphane or 6-HITC increased the number of HO-1-positive neurons, but did not affect the number of HO-1-positive astrocytes (Fig. 6E).

\section{HO-1 plays no role in the cytoprotection provided by sulforaphane and 6-HITC}

HO-1 oxidatively cleaves heme to biliverdin, forms $\mathrm{CO}$, and releases chelated $\mathrm{Fe}^{2+}$. Bilirubin, the reduction product of biliverdin, serves as a potent radical scavenger and protects against oxidative stress at nanomolar concentrations $(24,26,27)$. As sulforaphane and 6-HITC increased the expression of HO-1 in striatal cultures, we investigated the involvement of HO-1 in the neuroprotective effects. Zn-PP IX, a competitive HO-1 inhibitor, did not influence the protective effects of sulforaphane and 6-HITC on paraquat-induced cytotoxicity (Fig. 7: A, B). Furthermore, we examined the effect of bilirubin on oxidative stress. Bilirubin $(10-100 \mathrm{nM})$ did not affect $\mathrm{H}_{2} \mathrm{O}_{2}$ - or paraquat-induced cytotoxicity (Fig. 7: C, D).

\section{Discussion}

In the present study, we first examined the effect of isothiocyanates on the viability of rat striatal cultures. As shown in Fig. 1, sulforaphane and 6-HITC caused cell death at concentrations above $10 \mu \mathrm{M}$. These results are consistent with reports that isothiocyanates at high concentrations induced cell death in various types of cells (28). As a mechanism of cytotoxicity, isothiocyanates induced apoptosis by generating ROS and activating both intrinsic and extrinsic caspase cascades $(29,30)$. In our cultures, pretreatment with $0.01-1 \mu \mathrm{M}$ sulforaphane or $0.01-1 \mu \mathrm{M} 6-\mathrm{HITC}$ attenuated the cytotoxicity induced by $\mathrm{H}_{2} \mathrm{O}_{2}$ and paraquat (Fig. 2), whereas the co-application of sulforaphane and 6-HITC had no effect (data not shown). These results suggest that sulforaphane and 6-HITC activate intracellular defense systems against oxidative stress including the Nrf2-ARE pathway.

Nrf2, a member of the Cap'n' Collar family of basic leucine zipper proteins, was identified as a key transcription factor for ARE, a cis-regulating DNA sequence located in the promoter region of anti-oxidant enzymes and phase-II detoxifying enzymes (5). Under basal condi-
A
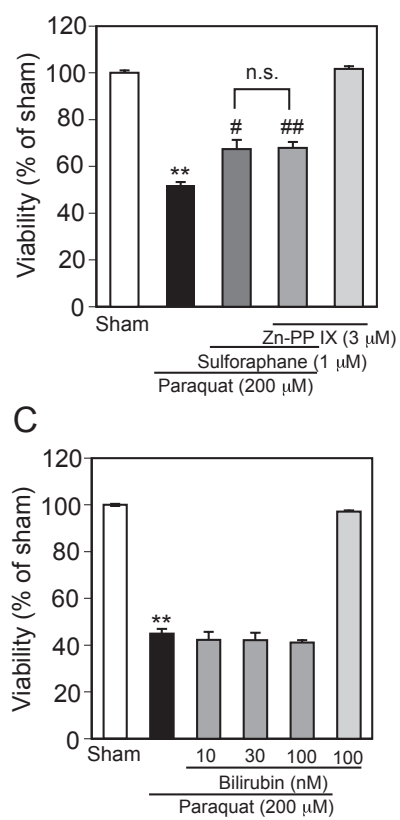

B

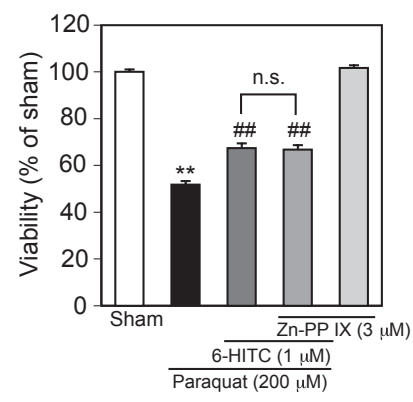

D

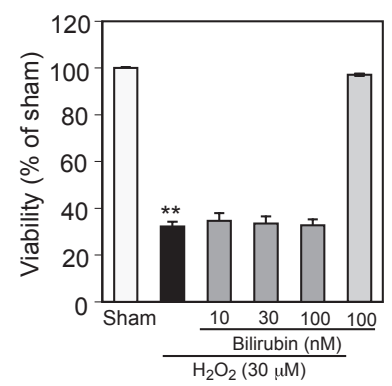

Fig. 7. HO-1 did not influence the protective effect of isothiocyanates on oxidative stress-induced cytotoxicity. A, B: ZnPP IX, a HO-1 inhibitor, was added with sulforaphane (A) and 6-HITC (B) for $24 \mathrm{~h}$, followed by $200 \mu \mathrm{M}$ paraquat for $24 \mathrm{~h}$. C, D: Cultures were pretreated with $10-100 \mathrm{nM}$ bilirubin for $1 \mathrm{~h}$ and simultaneously treated with $200 \mu \mathrm{M}$ paraquat (C) and $30 \mu \mathrm{M} \mathrm{H}_{2} \mathrm{O}_{2}$ (D) for $24 \mathrm{~h}$. ${ }^{* *} P<0.01$, compared with sham-treatment. ${ }^{\#} P<0.05,{ }^{\#} P<0.01$, compared with toxin alone. n.s.: not significant.

tions, Nrf2 is retained in the cytoplasm by Keap1. However, oxidative stress or electrophilic agents can disrupt the Nrf2/Keap1 complex and facilitate its translocation into the nucleus, where it enhances the transcription of ARE-driven genes, including the $\gamma$-GCS and HO-1 genes $(6,31)$. Recently, two mechanisms for activation of the Nrf2-ARE pathway by isothiocyanates including sulforaphane have been proposed. One is structural change of Keap1 due to the modification of specific cysteine residues caused by the binding of sulforaphane (32). The other is the phosphorylation of Nrf2 by ERK2, a mitogen-activated protein kinase, activated by sulforaphane (33). With either mechanism, the translocation of Nrf2 into the nucleus is important to induce the activation of the Nrf2-ARE pathway.

To clarify the neuroprotective mechanisms of isothiocyanates, we first focused on intracellular glutathione levels. Glutathione is important for cellular defense against ROS as an anti-oxidant in mammalian cells. It has been reported that the expression of $\gamma$-GCS, a limiting-enzyme in glutathione synthesis, was increased by the binding of Nrf2 to ARE $(5,20)$. Therefore, we examined the effect of isothiocyanates on the expression of 
$\gamma$-GCS and amount of glutathione in striatal cultures. Sulforaphane and 6-HITC increased the level of $\gamma$-GCS at $12 \mathrm{~h}$ in a concentration-dependent manner (Fig. 4A). Moreover, they increased significantly the total amount of intracellular glutathione after $24 \mathrm{~h}$. As shown in Fig. $5, \mathrm{GSH}$ and NAC, a precursor of glutathione synthesis, significantly prevented $\mathrm{H}_{2} \mathrm{O}_{2}$ - and paraquat-induced cell death in a concentration-dependent manner. Furthermore, co-treatment with $\mathrm{BSO}$, an irreversible inhibitor of $\gamma$-GCS, and isothiocyanates suppressed the protection of sulforapane and 6-HITC. These results suggested that an elevation of the intracellular glutathione levels plays a pivotal role in the cytoprotective effect of isothiocyanates.

In addition to increasing glutathione content, sulforaphane and 6-HITC enhanced the expression of HO-1, which peaked about $12 \mathrm{~h}$ after treatment (Fig. 6: A, B). HO-1, part of the heme oxygenase family, mediates heme catabolism in a wide range of species (24). Under normal conditions, HO-1 is barely detectable in the brain. However, immunoreactivity to HO-1 is increased in neurons and astrocytes in various neurodegenerative diseases such as Alzheimer's disease and Parkinson's disease (34). Several lines of evidence have indicated that transcriptional induction of HO-1 is also regulated by ARE $(24-26)$. As shown in Fig. 6: C, D, the induction of HO-1 expression was attenuated by simultaneous treatment with cycloheximide, which inhibits de novo protein synthesis. These results suggested that isothiocyanates would increase the expression of HO-1 through de novo synthesis via the Nrf2-ARE pathway.

It is reported that $\mathrm{HO}-1$ has protective effects against oxidative stress such as $\mathrm{H}_{2} \mathrm{O}_{2}(25,35)$. We examined the involvement of HO-1 in the protection provided by isothiocyanates. HO-1 catalyzes degradation of heme to yield biliverdin, free iron and carbon monoxide. Biliverdin is subsequently converted to bilirubin through the actions of biliverdin reductase (24). Bilirubin serves as a potent radical scavenger and protects against oxidative stress at nanomolar concentrations $(26,27)$. Unexpectedly, Zn-PP IX, a competitive inhibitor of HO-1, did not influence the protective effect of sulforaphane- and 6-HITC on paraquat-induced cytotoxicity. Furthermore, pretreatment with bilirubin had no effect on the cytotoxicity caused by $\mathrm{H}_{2} \mathrm{O}_{2}$ and paraquat. These findings suggested that it would be important for cytoprotection by isothiocaynates to elevate the amount of intracellular glutathione rather than to increase the expression of HO-1.

In the present study, we observed several differences in effect between sulforaphane and 6-HITC, including: a) the time taken to translocate Nrf2 into the nucleus, b) the intensity of $\gamma$-GCS expression, and c) the duration of
HO-1 expression. Moreover, 6-HITC was more potent than sulforaphane in inducing expression of $\gamma$-GCS and HO-1. Previous structure-activity studies have shown that 6-HITC induced greater activation of the Nrf2-ARE pathway than sulforaphane $(8,36)$. However, we could find no difference in the cytoprotective effect between sulforaphane and 6-HITC (Fig. 2). As a reason for these results, we consider that there was no difference in the effect on the intracellular glutathione content, which plays a pivotal role in the cytoprotection of isothiocyanates, between sulforaphane and 6-HITC.

In conclusion, we demonstrated that sulforaphane and 6-HITC, naturally occurring isothiocyanates, activated the intracellular antioxidant defense system through the Nrf2-ARE pathway and provided protection against the cytotoxicity induced by oxidative stress in CNS neurons. As oxidative stress is implicated in the etiology of many neurodegenerative disorders, isothiocyanates may be a potential tool for the prevention and treatment of such diseases.

\section{References}

1 Lin MT, Beal MF. Mitochondrial dysfunction and oxidative stress in neurodegenerative diseases. Nature. 2006;443:787795.

2 Droge W, Schipper HM. Oxidative stress and aberrant signaling in aging and cognitive decline. Aging Cell. 2007;6:361-370.

3 Blomgren K, Hagberg H. Free radicals, mitochondria and hypoxia-ischemia in the developing brain. Free Radic Biol Med. 2006; 40:388-397.

4 Trushina E, Mcmurray CT. Oxidative stress and mitochondrial dysfunction in neurodegenerative diseases. Neuroscience. 2007; 145:1233-1248.

5 MyzaK MC, Dashwood RH. Chemoprotection by sulforaphane: keep one eye beyond Keap1. Cancer Lett. 2006;233:208-218.

6 Itoh K, Wakabayashi N, Katoh Y, Ishii T, Igarashi K, Engel JD. Keap1 represses nuclear activation of antioxidant responsive elements by Nrf2 through binding to the amino-terminal Neh2 domain. Genes Dev. 1999;13:76-86.

7 Zhang Y, Talalay P, Cho CG, Posner GH. A major inducer of anticarcinogenic protective enzymes from broccoli: isolation and elucidation of structure. Proc Natl Acad Sci U S A. 1992;89: 2399-2403.

8 Morimitsu Y, Nakagawa Y, Hayashi K, Fujii H, Kumagai T, Nakagawa $Y$, et al. A sulforaphane analogue that potently activates the Nrf2-dependent detoxification pathway. J Biol Chem. 2002;277:3456-3463.

9 Matton MP, Cheng A. Neurohormetic phytochemicals: Low-dose toxins that induce adaptive neuronal stress responses. Trends Neurosci. 2006;29:632-639.

10 Misiewicz I, Skupinska K, Kowalska E, Lubinski J, Kasuprzycka GT. Sulforaphane-mediated induction of a phase 2 detoxifying enzyme NAD(P)H:quinone reductase and apoptosis in human lymphoblastoid cells. Acta Biochim Pol. 2004;51:711-721.

11 Gao X, Dinkova-kostova AT, Talalay P. Powerful and prolonged protection of human retinal pigment epithelial cells, keratino- 
cytes, and mouse leukemia cells against oxidative damage: the indirect antioxidant effects of sulforaphane. Proc Natl Acad Sci U S A. 2001;98:15221-15226.

12 Nomura T, Shinoda S, Yamori T, Sawaki S, Nagata I, Ryoyama $\mathrm{K}$, et al. Selective sensitivity to wasabi-derived 6-(methylsulfinyl)hexyl isothiocyanate of human breast cancer and melanoma cell lines studied in vitro. Cancer Detect Prev. 2005;29:155160.

13 Kraft AD, Johnson DA, Johnson JA. Nuclear factor E2-related factor 2-dependent antioxidant response element activation by tert-butylhydroquinone and sulforaphane occurring preferentially in astrocytes conditions neurons against oxidative insult. J Neurosci. 2004;24:1101-1112.

14 Zhao J, Kobori N, Aronowski J, Dash PK. Sulforaphane reduces infarct volume following focal cerebral ischemia in rodents. Neurosci Lett. 2006;393:108-112.

15 Zhao X, Sun G, Zhang J, Strong R, Dash PK, Kan YW, et al. Transcription factor Nrf2 protects the brain from damage produced by intracerebral hemorrhage. Stroke. 2007;38:3280-3286.

16 Osakada F, Hashino A, Kume T, Katsuki H, Kaneko S, Akaike A. Alpha-tocotrienol provides the most potent neuroprotection among vitamin $\mathrm{E}$ analogs on cultured striatal neurons. Neuropharmacology. 2004;47:904-915.

17 Yamamoto N, Sawada H, Izumi Y, Kume T, Katsuki H, Shimohama S, et al. Proteasome inhibition induces glutathione synthesis and protects cells from oxidative stress: relevance to Parkinson disease. J Biol Chem. 2007;282:4364-4372.

18 Day BJ, Shawen S, Liochev SI, Crapo JD. A metalloporphyrin superoxide dismutasemimetic protects against paraquat-induced endothelial cell injury, in vitro. J Pharmacol Exp Ther. 1995;275: 1227-1232.

19 Cocheme HM, Murphy MP. Complex I is the major site of mitochondrial superoxide production by paraquat. J Biol Chem. 2008; 283:1786-1798.

20 Shinkai Y, Sumi D, Fukami I, Ishii T, Kumagai Y. Sulforaphane, an activator of Nrf2, suppresses cellular accumulation of arsenic and its cytotoxicity in primary mouse hepatocytes. FEBS Lett. 2006;580:1771-1774.

21 Xu C, Yuan X, Pan Z, Shen G, Kim JH, Yu S, et al. Mechanism of action of isothiocyanates: the induction of ARE-regulated genes is associated with activation of ERK and JNK and the phosphorylation and nuclear translocation of Nrf2. Mol Cancer Ther. 2006;5:1918-1926.

22 Dringen R. Metabolism and functions of glutathione in brain. Prog Neurobiol. 2000;62:649-671.

23 Aoyama K, Watabe M, Nakaki T. Regulation of neuronal glutathione synthesis. J Pharmacol Sci. 2008;108:227-238.
24 Morse D, Choi AM. Heme oxygenase-1: the "emerging molecule" has arrived. Am J Respir Cell Mol Biol. 2002;27:8-16.

25 Li HY, Zhong YF, Wu SY, Shi N. NF-E2 related factor 2 activation and heme oxygenase-1 induction by tert-butylhydroquinone protect against deltamethrin-mediated oxidative stress in PC12 cells. Chem Res Toxicol. 2007;20:1242-1251.

26 Farrera JA, Jauma A, Ribo JM, Peire MA, Parellada PP, RoquesChoua $\mathrm{S}$, et al. The antioxidant role of bile pigments evaluated by chemical tests. Bioorg Med Chem. 1994;2:181-185.

27 Dore S, Takahashi M, Ferris CD, Zakhary R, Hester LD, Guastella D, et al. Bilirubin, formed by activation of heme oxygenase-2, protects neurons against oxidative stress injury. Proc Natl Acad Sci U S A. 1999;96:2445-2450.

28 Fimognari C, Hrelia P. Sulforaphane as a promising molecule for fighting cancer. Mutat Res. 2007;635:90-104.

29 Pham NA, Jacobberger JW, Schimmer AD, Cao P, Gronda M, Hedley DW. The dietary isothiocyanate sulforaphane targets pathways of apoptosis, cell cycle arrest, and oxidative stress in human pancreatic cancer cells and inhibits tumor growth in severe combined immunodeficient mice. Mol Cancer Ther. 2004;3:1239-1248.

30 Singh SV, Srivastava SK, Choi S, Lew KL, Antosiewicz J, Xiao $\mathrm{D}$, et al. Sulforaphane-induced cell death in human prostate cancer cells is initiated by reactive oxygen species. J Biol Chem. 2005;280:19911-19924.

31 Numazawa S, Yoshida T. Nrf2-dependent gene expressions: a molecular toxicological aspect. J Toxicol Sci. 2004;29:81-89.

32 Zhang DD, Hannink M. Distinct cysteine residues in Keap1 are required for Keap1-dependent ubiquitination of $\mathrm{Nrf} 2$ and for stabilization of Nrf2 by chemopreventive agents and oxidative stress. Mol Cell Biol. 2003;23:8137-8151.

33 Yu R, Lei W, Mandlekar S, Weber MJ, Der CJ, Wu J, et al. Role of a mitogen-activated protein kinase pathway in the induction of phase II detoxifying enzymes by chemicals. J Biol Chem. 1999; 274:27545-27552.

34 Schipper HM. Heme oxygenase expression in human central nervous system disorders. Free Radic Biol Med. 2004;37: 1995-2011.

35 Moon MK, Choi BM, Oh GS, Pae HO, Kim JD, Oh H, et al. Catalposide protects Neuro 2A cells from hydrogen peroxideinduced cytotoxicity via the expression of heme oxygenase-1. Toxicol Lett. 2003;145:46-54.

36 Hon DX, Fukuda M, Fujii M, Fuke Y. Transcriptional regulation of nicotinamide adenine dinucleotide phosphate: quinone oxidoreductase in murine hepatoma cells by 6-(methylsufinyl) hexyl isothiocyanate, an active principle of wasabi (Eutrema wasabi Maxim). Cancer Lett. 2000;161:195-200. 